\title{
Molecular mechanism underlying miR-130b-Sp1 transcriptional regulation in LPS-induced upregulation of MUC5AC in the bile duct epithelium
}

\author{
XIAODONG WU, CHENHUI YAO, JING KONG, YU TIAN, YING FAN, \\ ZHEN ZHANG, JINYAN HAN and SHUODONG WU \\ Department of Secondary General Surgery, Shengjing Hospital of China Medical University, \\ Shenyang, Liaoning 110004, P.R. China
}

Received January 4, 2020; Accepted October 16, 2020

DOI: $10.3892 / \mathrm{mmr} .2020 .11745$

\begin{abstract}
Hepatolithiasis is a common disease that represents a serious health threat to the Chinese population. The pathological mechanism underlying hepatolithiasis is closely related to bacterial infections of the intrahepatic bile duct, followed by chronic inflammation and the overexpression of mucin $5 \mathrm{AC}$ (MUC5AC). However, the exact mechanism responsible for the lipopolysaccharide (LPS)-induced upregulation of MUC5AC has yet to be elucidated. Specificity protein 1 (Spl) is a ubiquitous transcription factor that plays a vital role in the regulation of a number of genes that are responsible for normal cellular function. microRNA (miR/miRNA)-130b is a member of the miRNA family. miRNAs can bind to the 3'-untralsated region (3'-UTR) of a target gene and influence its expression levels. The present study found that LPS increases the expression of MUC5AC by influencing Sp1 secretion. Chromatin immunoprecipitation-quantitative PCR experiments further verified three Spl binding sites in the MUC5AC promoter sequence that can regulate the expression of MUC5AC. Further analysis demonstrated that Sp1 expression was regulated by miR-130b. Luciferase experiments identified one miR-130b binding site in the Sp1 3'-UTR region. In vivo experiments also confirmed the role of the miR-130b-Sp1-MUC5AC signaling pathway in the formation of biliary stones and indicated that this pathway may provide targeted therapeutic strategies for the treatment of intrahepatic bile duct stones.
\end{abstract}

\section{Introduction}

Hepatolithiasis is a common disease that poses a serious health threat to the Chinese population, with an incidence rate of

Correspondence to: Dr Shuodong Wu, Department of Secondary General Surgery, Shengjing Hospital of China Medical University, 36 Sanhao Street, Heping, Shenyang, Liaoning 110004, P.R. China E-mail: wushuodong2019@163.com

Key words: hepatolithiasis, mucin $5 \mathrm{AC}$, specificity protein 1, microRNA-130b
$2-25 \%$ (1). The underlying pathology of hepatolithiasis involves chronic proliferative cholangitis of the mucus-producing major intrahepatic bile duct; this represents the key lesion associated with this condition $(2,3)$. The proliferative glands and biliary epithelium produce high amounts of mucin to initiate the process of stone formation and development, which comprise calcium salts and lipids (4). This is an important pathological mechanism that can lead to chronic proliferative cholangitis and stone formation (5). Bacterial infection within the biliary system is closely related to the development of cholelithiasis, in which Gram-negative bacteria, including Escherichia coli and Klebsiella, produce lipopolysaccharides (LPS), a common substance in infectious bile and gallstone cores $(6,7)$. A total of 22 mucin genes have been identified to date (8). Of these, mucin 5AC (MUC5AC) has been identified as the most important mucin secreted by the bile duct $(9,10)$. This protein is expressed at high levels in patients with primary hepatolithiasis and is an important nuclear-promoting factor (11). Previous studies from our research group reported that under normal conditions, MUC5AC is only expressed at low levels in the human intrahepatic bile duct (12). However, the expression of MUC5AC is known to significantly increase following LPS treatment, and there is a positive association between MUC5AC expression and LPS concentration (13). At present, studies on the regulatory mechanisms underlying the overexpression of MUC5AC in response to a variety of factors have primarily focused on the airway epithelium and nasal mucosa (10). The regulation of MUC5AC expression is a result of the enhanced transcription of the MUC5AC gene; however, little is known about such mechanisms in the biliary system (14). Therefore, it is important to elucidate the specific mechanisms underlying mucin synthesis in the bile duct epithelium to develop strategies to inhibit the oversecretion of bile duct mucus. This would create a new therapeutic target for hepatolithiasis and thus help to prevent and treat this disease.

The transcription initiation site of the MUC5AC gene is located 48 base pairs (bp) upstream of the translation initiation codon (15). There is a sequence $\sim 1,000 \mathrm{bp}$ upstream of the initiation codon that features a dense distribution of transcription factor binding sites; these binding sites constitute the primary area that regulates the expression of the MUC5AC 
gene (15). This region regulates MUC5AC in a manner that shows differential expression in different tissues and cells; this occurs via the combined action of different transcription factors (9). Specificity protein 1 (Sp1), a member of the SP family, is a ubiquitously expressed transcription factor (7). Sp1 contains a zinc finger domain at the $\mathrm{C}$ terminal, which can specifically recognize the GC box element in DNA sequences and participate in the transcriptional regulation of various genes, such as vascular endothelial growth factor (VEGF) and human epidermal growth factor receptor 2 (HER-2) $(16,17)$. Studies have demonstrated that $\mathrm{Sp} 1$ is the primary regulator of MUC5AC expression, and Sp1 expression can directly activate the promoter of MUC5AC, induce MUC5AC gene transcription and promote the high expression of MUC5AC in mucin (15). Electrophoretic mobility shift assays revealed that following activation, $\mathrm{Spl}$ protein translocates into the nucleus and binds to the regulatory element corresponding to the MUC5AC gene promoter, which is the main targeting element for MUC5AC transcription (16). Other previous studies have demonstrated that even in the same tissue, Sp1 expression induced by various stimuli is not consistent at the binding site of control elements in the MUC5AC promoter $(15,18)$. A previous study reported that the $\mathrm{Spl}$ binding site within the 324-64 bp sequence of the MUC5AC 5'-upstream region is an important regulatory element for neutrophil elastase-induced MUC5AC gene expression (19). Another study investigating cigarette smoking-induced secretion of MUC5AC from epithelial cells in the airway revealed that smoking induced $\mathrm{Sp} 1$ protein expression, phosphorylation, translocation to the nucleus and binding to the MUC5AC promoter-3724/-3224 bp corresponding sequences, indicating that smoking can induce high expression of MUC5AC; while the rest of the original cis-element exerted no such function (18). The present study focused on a regulatory role for $\mathrm{Sp} 1$ in transcription and the binding site of Sp1 and the MUC5AC promoter in LPS-induced overexpression of MUC5AC in the bile duct epithelium. This represents the primary focus of the current study.

Further studies on the regulation of Sp1 expression found that in LPS-treated RAW264.7 macrophages, microRNA (miRNA/miR)-130b can directly interact with the 3'-untranslated region (3'-UTR) of the Sp1 gene and inhibit the expression of Sp1 (20), suggesting that the expression of Sp1 is regulated by miR-130b. miRNAs are a class of small non-coding s RNA molecules $(21,22)$ that can specifically bind to the 3'-UTR of the target mRNA by complementary pairing, which blocks gene expression by degrading or inhibiting the target gene mRNA $(23,24)$. Abnormal miRNA expression can lead to the disturbance of the corresponding regulatory network and represents an important factor underlying the occurrence of diseases $(25,26)$. Therefore, determining whether the regulation of Spl expression is mediated by miR-130b during the overexpression of MUC5AC in the LPS-induced bile duct epithelium formed the second aim of the current study.

\section{Materials and methods}

Ethics. All animal studies were approved by the local ethics committee of the Affiliated Shengjing Hospital of China Medical University (approval no. 2017PS231K). All animals were maintained under 12-h light/dark cycles and had free access to food and water at $25^{\circ} \mathrm{C}$ and normal atmospheric pressure. All handling procedures aimed to minimize suffering and conformed to the standards of the Ethics Committee of Shengjing Hospital.

Cell lines and cell culture. Human intrahepatic biliary epithelial cells (HIBEpiCs) were purchased from ScienCell Research Laboratories, Inc., and cultivated in epithelial cell medium containing $500 \mathrm{ml}$ basal epithelium medium (ScienCell Research Laboratories, Inc.), $5 \mathrm{ml}$ epithelial cell growth supplement (ScienCell Research Laboratories, Inc.), $10 \mathrm{ml}$ fetal bovine serum (ScienCell Research Laboratories, Inc.) and $5 \mathrm{ml}$ penicillin/streptomycin solution (ScienCell Research Laboratories, Inc.) in an incubator at $37^{\circ} \mathrm{C}$ with $5 \% \mathrm{CO}_{2}$. The cell medium was refreshed every 1-2 days, and passaged at a ratio of $1: 2$ or $1: 3$ every 3 to 5 days.

\section{Cell pretreatment and administration of LPS and inhibitors. To} investigate the effect of LPS exposure, HIBEpiCs were treated with different concentrations of LPS (1, 10 and $100 \mu \mathrm{g} / \mathrm{ml}$ and a negative control, which was without LPS exposure) for $24 \mathrm{~h}$ once cells have reached $60-70 \%$ confluency. Mithramycin A (MA; $10 \mu \mathrm{g} / \mathrm{ml}$; Sigma-Aldrich; Merck KGaA), an Sp1 inhibitor, was used for inhibitory experiments. HIBEpiCs were incubated with $1 \mu \mathrm{g} / \mathrm{ml} \mathrm{MA}$ and LPS for $24 \mathrm{~h}$ at $37^{\circ} \mathrm{C}$.

Western blotting. Protein expression was measured by western blotting, as described previously $(3,6)$, with some modifications. The precipitate protein was lysed in lysis buffer (Beyotime Institute of Biotechnology), and protein concentrations were measured using a Bradford protein assay kit (Bio-Rad Laboratories, Inc.). Equal amounts of protein (15 $\mu \mathrm{g})$ were separated via SDS-PAGE with an $8 \%$ gel and then transferred onto polyvinylidene difluoride membranes. Membranes were subsequently blocked with $5 \%$ nonfat milk in TBS with $0.1 \%$ Tween-20 (TBS-T) at room temperature for $1.5 \mathrm{~h}$ to eliminate non-specific binding. Membranes were then incubated overnight with primary antibodies against Sp1 (1:1,000; cat. no. ab124804; Abcam) and GADPH (1:3,000; cat. no. 60004-1-Ig; ProteinTech Group, Inc.) at $4^{\circ} \mathrm{C}$. Subsequently, membranes were washed three times in TBS-T and incubated with secondary antibodies $2 \mathrm{~h}$ at room temperature (horseradish peroxidase-conjugated anti-rabbit immunoglobulin G; 1:3,000; cat. nos. WB0177 and A23210; Beijing Zhongshan Golden Bridge Biotechnology Co., Ltd.). Immunoreactive bands were then visualized using an enhanced chemiluminescent kit (EMD Millipore) according to the manufacturer's instructions. Band densities were quantified using Gel-Pro Analyzer densitometry software (version 6.3; Media Cybernetics, Inc.

Reverse transcription-quantitative PCR (RT-qPCR). Total RNA was extracted from HIBEpiCs cell cultures using TRIzol ${ }^{\circledR}$ (Invitrogen; Thermo Fisher Scientific, Inc.). RNA concentration was determined by spectrophotometry. RT-qPCR was performed using the PrimeScript RT reagent kit according to the manufacturer's instructions with gDNA Eraser (Takara Bio, Inc.) and SYBR Green Premix Ex Taq (Takara Bio, Inc.). RT-qPCR was performed as follows: $10 \mathrm{~min}$ at $95^{\circ} \mathrm{C}$, followed by 35 cycles of $15 \mathrm{sec}$ at $95^{\circ} \mathrm{C}$ and $40 \mathrm{sec}$ at $55^{\circ} \mathrm{C}$. The following primer pairs were used for the qPCR: MUC5AC, forward, 
5'-AGCCGGCAACCTACTACTCG-3' and reverse, 5'-AAG TGGTCATAGGCTTCGTGC-3'; Sp1 forward, 5'-TGGCAG CAGCAGTACCAATGGC-3' and reverse, 5'-CCAGGTAGT CCTGTCAGAACTT-3'; miR-130b-3p CAGUGCAAUGAU GAAAGGGCAU-polyA; GADPH forward, 5'-ACAACTTTG GTATCGTGGAAGG-3' and reverse, 5'-GCCATCACGCCA CAGTTTC-3' and U6, forward, 5'-GGAACGATACAGAGA AGATTAGC-3' and reverse, 5'-TGGAACGCTTCACGAATT TGCG-3'. Relative expression levels were calculated using the $2^{-\Delta \Delta \mathrm{Ca}}$ method (6) following normalization to the expression of GAPDH or U6.

Immunohistochemistry (IHC). IHC was performed to detect the expression of MUC5AC in bile duct tissue obtained from clinical specimens. Tissues were obtained from patients who underwent partial hepatectomy due to intrahepatic bile duct stones in the General Surgery Department of Shengjing Hospital between September 2017 and November 2018; these tissues acted as an experimental group. Normal adjacent tissue, obtained from hepatic hemangioma resection surgery, were selected as the control group. The sample collecting procedure was approved by the local ethics committee of the Affiliated Shengjing Hospital of China Medical University (approval no. 2017PS231K). All patients provided written informed consent. The bile duct tissue was fixed overnight in $4 \%$ paraformaldehyde at $4{ }^{\circ} \mathrm{C}$, sectioned into $4-\mu \mathrm{m}$ slices. To block endogenous peroxidase activity, each section was incubated with $0.3 \% \mathrm{H}_{2} \mathrm{O}_{2}$ at room temperature for $10 \mathrm{~min}$. After blocking in 5\% bovine serum albumin (Beijing ZSGB-BIO Technology, Ltd.) for $20 \mathrm{~min}$ at room temperature, the sections were incubated with primary MUC5AC antibody (1:1,000; cat. no. ab3649; Abcam) overnight at $4^{\circ} \mathrm{C}$. The next day, sections were washed three times using PBS, then incubated with biotin-conjugated anti-mouse IgG (1:100; cat. no. BM2004; Wuhan Boster Biological Technology, Ltd.) secondary antibody for $20 \mathrm{~min}$ at room temperature. Lastly, the sections were incubated with HRP-streptavidin (1:1,000; cat. no. BIR701-3; Beijing Borsi Technology Co.,Ltd.) at room temperature for $10 \mathrm{~min}$, then visualized using a DAB peroxidase kit (1:20; cat. no. AR1000; Wuhan Boster Biological Technology, Ltd.) at room temperature for $1 \mathrm{~min}$, then counterstained with hematoxylin at room temperature for $3 \mathrm{~min}$. The stained slides were visualized under light microscope (magnification, x200 and x400; Eclipse Ci-L; Nikon Corporation) and the images were analyzed using ImageJ $1.51 \mathrm{~K}$ (National Institutes of Health). Quantitative analysis was completed based on the percentage of positive cells with MUC5AC staining (0-25\%, low; 25-50\%, medium; $>50 \%$, high). The details of clinical specimens were shown in Table I.

\section{Immunofluorescence (IF)}

Tissue IF. Fluorescent co-staining experiments were performed to verify the association between MUC5AC expression (cat. no. ab3649; Abcam) and Sp1 expression (cat. no. ab124804; Abcam) in bile duct tissue. IF was performed as the aforementioned IHC procedure. Tissues were incubated with fluorescent secondary antibody (1:100; cat. no. FITC-10835; ProteinTech Group, Inc.). Subsequently, sections were incubated with DAPI for $5 \mathrm{~min}$ to stain cell nuclei. The stained slides were visualized under fluorescence microscope (magnification, x200 and $x 400$; Eclipse Ci-L; Nikon Corporation) and the images were analyzed using Image J $1.51 \mathrm{~K}$ (National Institutes of Health). Quantitative analysis was completed based on the percentage of positive cells with MUC5AC and Sp1 staining (0-25\%, low; $25-50 \%$, medium; $>50 \%$, high).

Cell IF. HIBEpiCs pretreated with $100 \mu \mathrm{g} / \mathrm{ml}$ of LPS for $24 \mathrm{~h}$ were used as the experimental group for cell IF analysis. Variation in MUC5AC and Sp1 expression were then compared between experimental and negative control groups. Following LPS treatment, cultured HIBEpiCs were fixed with $-20^{\circ} \mathrm{C}$ methanol for $10 \mathrm{~min}$, washed in PBS three times for 5 min, then blocked with 1\% BSA (cat. no A8020; Beijing Solarbio Science \& Technology Co., Ltd.) in PBS for $30 \mathrm{~min}$ at room temperature. Following washing in PBS three times for $5 \mathrm{~min}$, the sections were then simultaneously incubated with MUC5AC (cat. no. ab3649) and Sp1 (cat. no. ab124804) primary antibodies at 1:100 dilution (Abcam) overnight at $4^{\circ} \mathrm{C}$. Sections were then washed in PBS three times for $5 \mathrm{~min}$ and incubated in fluorescent secondary antibody (goat anti-rat; cat. no. SA00009-1 and goat anti-rabbit; cat. no. FITC-10835; both 1:200; ProteinTech Group, Inc.) for $1 \mathrm{~h}$ at room temperature. Nuclei were then stained with DAPI for $5 \mathrm{~min}$ at room temperature.

Enzyme-linked immunosorbent assay (ELISA). ELISA was used to detect the concentration of MUC5AC in HIBEpiCs cell supernatants, which was produced by collecting and centrifuging cells in an ultrafiltration tube (EMD Millipore) at $2,000 \mathrm{x} \mathrm{g} 5 \mathrm{~min}$ at room temperature. Subsequently, $250 \mu \mathrm{l}$ concentrated cell supernatant solution was obtained from the control, LPS exposure, Sp1 shRNA transfection and miR130b mimics intervention groups and assayed using the human MUC5AC ELISA assay kit (cat. no. CSB-E10109h; Cusabio Technology, LLC) to determine MU5AC concentration.

Cell transfection. Short hairpin RNA (shRNA) plasmids were for the specific inhibition of Sp1 and miR-130b mimic and inhibitor were purchased from Shanghai GeneChem Co., Ltd. A total of $8 \mu \mathrm{g}$ shRNA plasmids and miR-130b mimic were transfected into HIBEpiCs using Lipofiter Liposomal transfection reagent (Hanbio Biotechnology Co., Ltd.) for $48 \mathrm{~h}$ before experimentation. Transfection efficiency of shRNA efficiency was measured by RT-qPCR and western blotting. The sequences of the shRNAs and miRs used are as follows: Sp1 overexpression plasmids (cat, no. 51288; Genechem Co., Ltd.), 5'-AGAAGGAGAGCAAAACCAGC-3'; Sp1 suppression shRNA (cat, no. 4090; Genechem Co., Ltd.), 5'-CCTGGT GCAAACCAACAGATT-3'; miR-130b mimic (cat, no. 14159; Genechem Co.,Ltd.),5'-ATGCCCTTTCATCATTGCACTG-3' and miR-130b inhibitor (cat. no. 18930; Genechem Co., Ltd.), 5'-ATGCCCTTTCATCATTGCACTG-3'.

Luciferase reporter gene assay. Luciferase reporter gene assays were performed to verify the direct binding effect and exact binding position between miR-130b and Sp1 3'-UTR sequences. The binding site was predicted using an online tool (http://www.targetscan.org). Plasmids (Genechem Co., Ltd.) for miR-130b, the Sp1 3'-UTR and mutant Sp1 3'-UTR (3'UTR-mu were constructed (Table II). miR-130b was then co-transfected by Nanofectin transfection reagent (PAA 
Table I. The details of clinical specimens.

\begin{tabular}{lcc}
\hline Variable & $\begin{array}{c}\text { Control group } \\
\mathrm{n}=8\end{array}$ & $\begin{array}{c}\text { Patients with hepatoliths } \\
\mathrm{n}=10\end{array}$ \\
\hline Age, years & $52 \pm 16$ & $61 \pm 24$ \\
Male (n, \%) & $5(62.5 \%)$ & $6(60 \%)$ \\
Female (n, \%) & $3(37.5 \%)$ & $4(40 \%)$ \\
\hline
\end{tabular}

Laboratories GmbH; GE Healthcare) with Sp1 3'-UTR and Sp1 3'UTR-mu into HIBEpiCs. Luciferase activities were then detected by a Dual-Luciferase Reporter Assay system (Promega Corporation) $48 \mathrm{~h}$ after transfection. The data were quantified by normalizing to Renilla luciferase activity.

Chromatin immunoprecipitation (ChIP) assay. Human HIBEpiCs were fixed with $1 \%$ formaldehyde for $15 \mathrm{~min}$ at room temperature and quenched with $0.125 \mathrm{M}$ glycine. Chromatin was then isolated using lysis buffer (Active Motif, Inc.). Lysates were sonicated using the EpiShear ${ }^{\mathrm{TM}}$ Probe Sonicator (Active Motif, Inc.) with an EpiShear ${ }^{\mathrm{TM}}$ Cooled Sonication Platform (Active Motif, Inc.), and the DNA was sheared to an average length of 300-500 bp. Genomic DNA was then prepared by treating aliquots of chromatin with RNase, proteinase $\mathrm{K}$ and heating for decrosslinking overnight at $65^{\circ} \mathrm{C}$, followed by purification using the QIAquick PCR Purification kit (Qiagen China Co., Ltd.). The resultant DNA was then quantified on a NanoDrop ${ }^{\mathrm{TM}}$ spectrophotometer (Thermo Fisher Scientific, Inc.). Extrapolation to the original chromatin volume allowed quantitation of the total chromatin yield. Genomic DNA regions of interest were then isolated using antibodies against Sp1 (1:150; cat, no. ab231778; Abcam). Complexes were eluted from the beads with SDS buffer and subjected to RNase and proteinase $\mathrm{K}$ treatment $(10 \mathrm{mg} / \mathrm{ml})$ at $45^{\circ} \mathrm{C}$. Crosslinks were reversed by overnight incubation with $5 \mathrm{M} \mathrm{NaCl}$ at $65^{\circ} \mathrm{C}$, and Chip DNA was purified using a QIAquick PCR Purification kit (Qiagen China Co., Ltd.). qPCR reactions were performed in triplicate using TB Green ${ }^{\mathrm{TM}}$ Premix Ex Taq $^{\mathrm{TM}}$ (Takara Bio, Inc.) on a CFX Connect ${ }^{\mathrm{TM}}$ Real-Time PCR system (Bio-Rad Laboratories, Inc.). Two positive and two negative control sites were tested, plus three test sites in one gene of interest. The resultant signals were normalized for primer efficiency by performing QPCR for each primer pair using input DNA (pooled unprecipitated genomic DNA from each cell sample). Primer sequences for chromatin immunoprecipitation-quantitative PCR were shown in Table III.

Rat lithogene model. A total of 30 6-week-old male Sprague-Dawley rats (body weight $220 \pm 15$ g) were purchased from Beijing Dingguo Changsheng Biotechnology Co., Ltd. A total of 18 Sprague-Dawley rats were randomly divided into two groups ( $\mathrm{n}=9$ for control group and $\mathrm{n}=9$ for experiment group): Pseudo-operation control group and experimental group. The rats were first anesthetized with pentobarbital sodium (intraperitoneal injection, $30 \mathrm{mg} / \mathrm{kg}$ ), underwent a laparotomy and a polyethylene (PE) tube was inserted into their common bile duct and fixed. Rats were then injected with the appropriate drugs $(5 \mathrm{mg} / \mathrm{ml}$ LPS for the experimental group and distilled water
Table II. Primers used for the luciferase reporter gene assay.

\begin{tabular}{ll}
\hline Primer & \multicolumn{1}{c}{ Sequence } \\
\hline miR-130b-F & 5'-TGTGGAAAGGACGCGGGAT \\
& CGCCCCAGCCAGCCTGCATTC-3' \\
miR-130b-R & 5'-CAGCGGTTTAAACTTAAGCT \\
& AAAAAACACTTACCCTCTGG-3' \\
Sp1 3'UTR-F & 5'-GAGGAGTTGTGTTTGTGGAC-3' \\
Sp1 3'UTR-R & 5'-GACGATAGTCATGCCCCGCG-3' \\
Sp1 3'UTR-mut-F & 5'-GAGGAGTTGTGTTTGTGGAC-3' \\
Sp1 3'UTR-mut R & 5'-GACGATAGTCATGCCCCGCG-3' \\
\hline
\end{tabular}

Sp1, specificity protein 1. F, forward; R, reverse.

Table III.Primer sequences for chromatin immunoprecipitationquantitative PCR.

\begin{tabular}{lc}
\hline Binding site & \multicolumn{1}{c}{ Primer sequence } \\
\hline Sp1 binding & F: 5'-CACTCCTGCCACATGTGAAG-3' \\
site A & R: 5'-GTTCTGAGCACTTGCTTCCA-3' \\
Sp1 binding & F: 5'-TCAGGAGACAGAAGCAGG-3' \\
site B & R: 5'-TGAGGAGTGAGTGAGCCAAG-3' \\
Sp1 binding & F: 5'-TCGGAAACTGGGCTCTATCC-3' \\
site C & R: 5'-CCCTCAGCAGCCTCTGAGGA-3'
\end{tabular}

Sp1, specificity protein $1 ; \mathrm{F}$, forward; R, reverse.

for the control group) via the PE tubes over a period of 3 days. The pseudo-operation control group was injected with distilled water $(100 \mu \mathrm{l})$ and the experimental group was injected with LPS $(5 \mathrm{mg} / \mathrm{kg})$, at a total volume of $100 \mu \mathrm{l}$. The PE tubes were closed for $12 \mathrm{~h}$ after drug injection and then re-opened. The common bile duct and intrahepatic bile duct were harvested 15 days after surgery. The rats were anesthetized with pentobarbital sodium (intraperitoneal injection, $60 \mathrm{mg} / \mathrm{kg}$ ) and then sacrificed by cervical dislocation while bile duct samples were obtained. The expression levels of MUC5AC, Sp1 and miR-130b were evaluated by western blotting, RT-qPCR and IHC. No rats died during the modeling period.

Rat biochemical analysis. Blood samples were taken from each group of rats and analyzed for serum total bilirubin, ALT and AST levels using a biochemical analyzer (cat. no. 98-11084-01; cat. no. 98-24010-US; cat. no. 98-24016-US; Catalyst One; IDEXX).

Statistical analysis. Data are presented as the mean \pm SD. All figures were created using SPSS software (version 20.0; SPSS Inc.). Data were analyzed using one-way ANOVA and Tukey's post hoc test for multiple group comparisons. Pearson algorithm for bivariate correlation analysis was used in positive distribution data. Pearson of Spearman algorithm for bivariate correlation analysis was used in nonpositive distribution data. $\mathrm{P}<0.05$ was considered to indicate a statistically significant difference. 
A

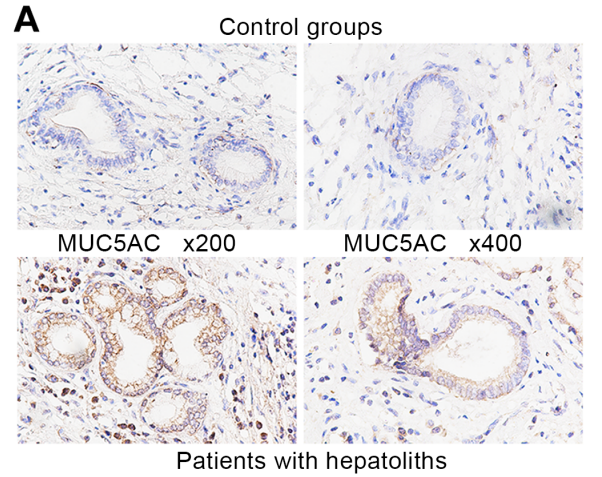

B
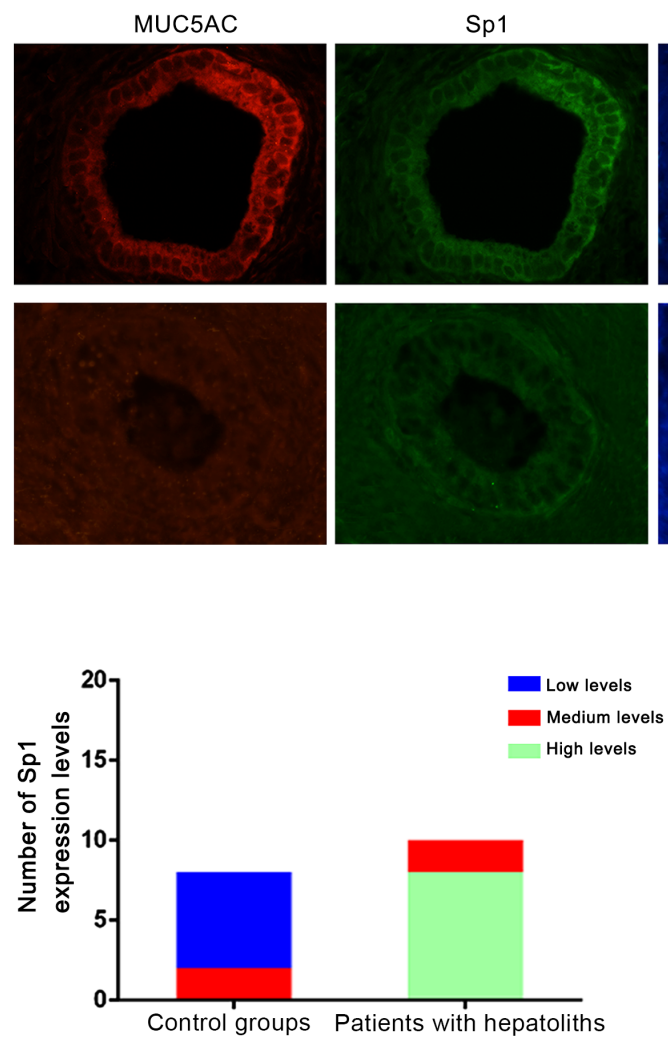
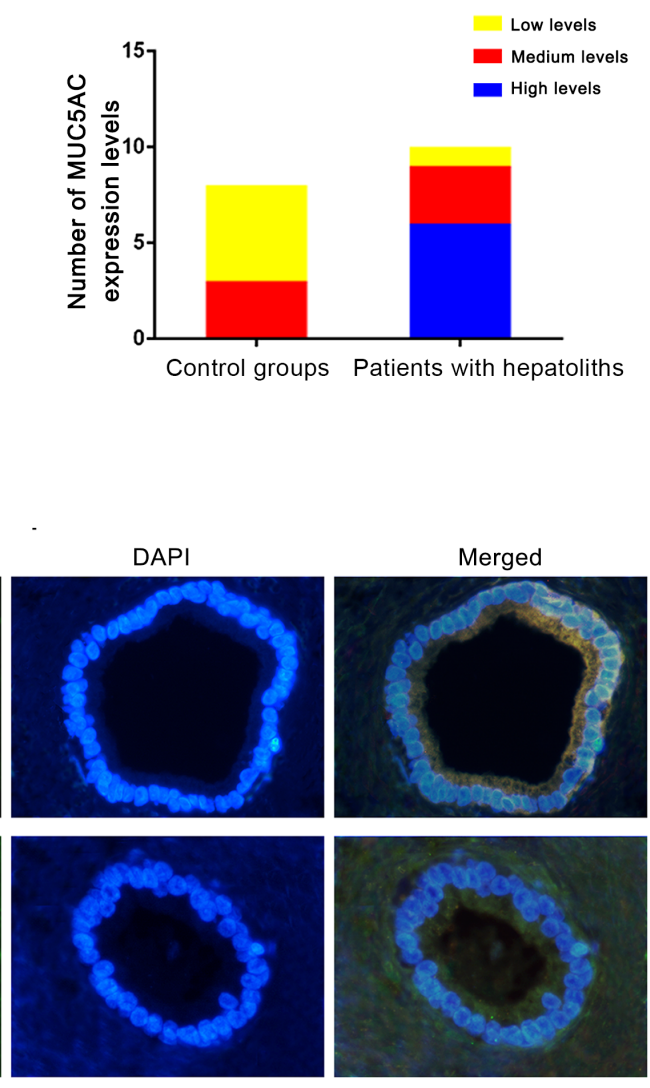

C

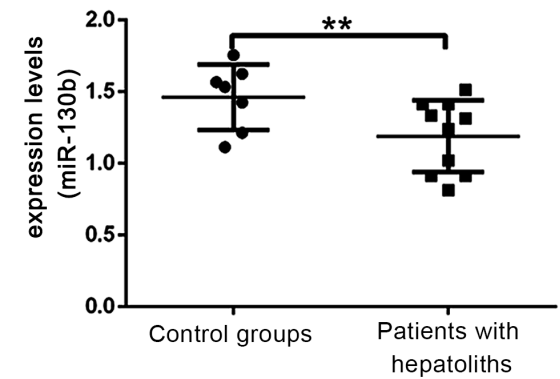

Figure 1. Detection of MUC5AC expression in normal bile duct tissue and in tissues from patients with hepatoliths. (A) Immunohistochemistry assay of MUC5AC staining in paraffin-embedded sections from normal human subjects and patients with hepatoliths at different magnifications and quantitative analysis of MUC5AC expression level of each section. (B) Co-staining immunofluorescence experiments for MUC5AC and Sp1 expression in sections from normal human subjects and patients with hepatoliths (magnification, $\mathrm{x} 400$ ), with quantitative analysis of Sp1 expression levels in each section. (C) Reverse transcription-quantitative PCR experiments for miR-130b expression in clinical tissue from normal human subjects and patients with hepatoliths. $\mathrm{n}=8$ in the control group; $\mathrm{n}=10$ in the hepatolith group. ${ }^{* *} \mathrm{P}<0.01$. MUC5AC, mucin 5AC; Sp1, specificity protein 1; miR, micro130b RNA.

\section{Results}

Expression levels of miR-130b, Spl and MUC5AC differed between intrahepatic cholangiolithiasis tissue and normal tissue. Bacterial infection of the biliary tract produces LPS, which then induces increased expression of MUC5AC $(2,3,6)$. The aim of the present study was to identify the underlying mechanism of this through a series of experiments. First, an IHC assay was performed to stain and compare the expression of MUC5AC in clinical paraffin-embedded sections from an intrahepatic cholangiolithiasis group and a control group. Based on the IHC results, MUC5AC staining was markedly higher in the intrahepatic cholangiolithiasis group compared with the control group (Fig. 1A). The regulatory function of $\mathrm{Sp} 1$ on MUC5AC expression and the effect of miR-130b on Sp1 expression, has been described briefly for diseases in other body systems $(15,18,19)$; however, their effects in biliary tract diseases is unknown. Therefore, MUC5AC and Sp1 were stained in clinical paraffin sections using an IF assay. Sp1 expression was higher in the intrahepatic cholangiolithiasis compared with the control group (Fig. 1B). The expression of miR-130b was significantly lower (Fig. 1C) in the cholangiolithiasis group compared with the control group. These findings suggested that miR-130b and Sp1 expressional changes may 
A

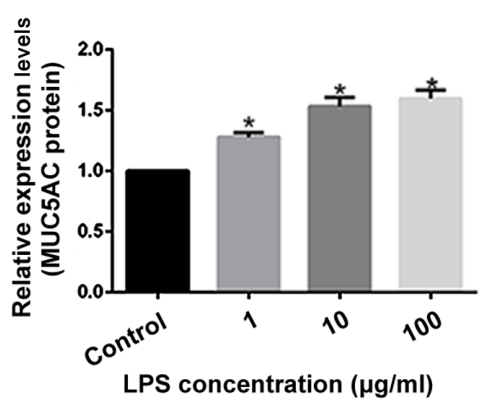

D

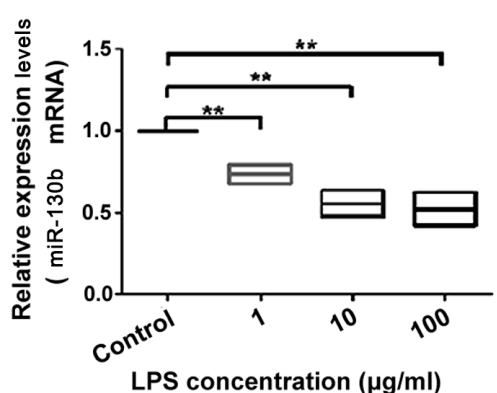

B

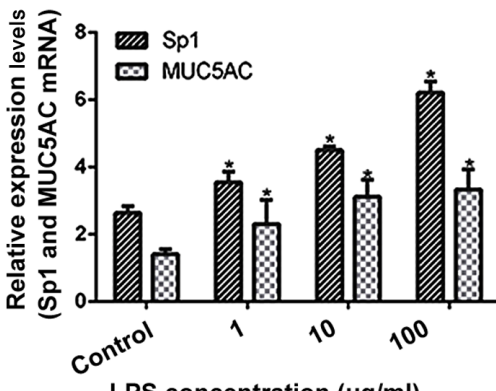

LPS concentration $(\mu \mathrm{g} / \mathrm{ml})$
C

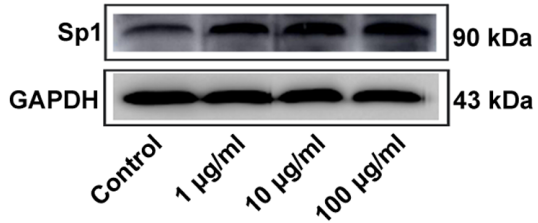

E

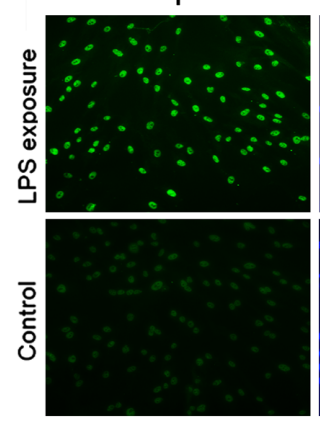

DAPI

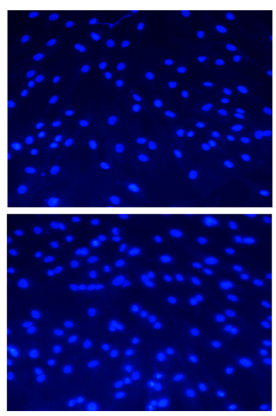

Merged

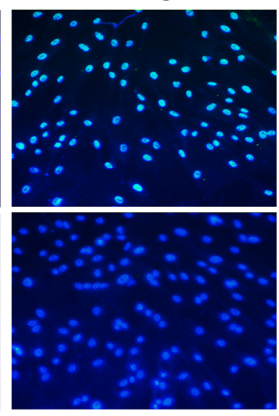

Figure 2. Detection of miR-130b, Sp1 and MUC5AC expression in HIBEpiCs following LPS treatment. (A) ELISA was performed to detect changes in MUC5AC expression in HIBEpiC supernatant. (B) RT-qPCR was performed to detect changes in MUC5AC and Sp1 mRNA expression in HIBEpiCs. (C) Western blotting was performed to detect changes Sp1 protein expression in HIBEpiCs. (D) RT-qPCR was performed to detect changes in miR-130b expression in HIBEpiCs. (E) immunofluorescence experiments for HIBEpiCs were performed to detect the transmembrane location of Sp1 in the control group compared with the $10 \mu \mathrm{g} / \mathrm{ml}$ LPS treatment group. Magnification, $\mathrm{x} 40 .{ }^{*} \mathrm{P}<0.05,{ }^{* *} \mathrm{P}<0.01$. MUC5AC, mucin 5AC; Sp1, specificity protein 1 ; miR, microRNA; HIBEpiCs, human intrahepatic biliary epithelial cells; LPS, lipopolysaccharide; RT-qPCR, reverse transcription-quantitative PCR.

be associated with MUC5AC changes and the occurrence of intrahepetic gallstones.

LPS induces the expression of MUC5AC, Spl and miR-130b in HIBEpiCs in a concentration-dependent manner. HIBEpiCs were incubated with of 1,10 and $100 \mu \mathrm{g} / \mathrm{ml}$ LPS for $24 \mathrm{~h}$. Using ELISA and RT-qPCR, it was found that the secretion of MUC5AC in the cell supernatant (Fig. 2A), and the expression of MUC5AC mRNA (Fig. 2B) significantly increased after LPS intervention in a concentration-dependent manner. Similarly, Sp1 mRNA (Fig. 2B) and protein expression levels (Fig. 2C) increased in HIBEpiCs following LPS interference in a concentration-dependent manner. Meanwhile, miR-130b levels significantly decreased upon increasing LPS concentration (Fig. 2D). The Sp1 DNA-binding effect in LPS-induced cell nuclei was also higher compared with the control group (Fig. 2E). Thus, changes in MUC5AC expression in the intrahepatic cholangiolithiasis biliary tissue after LPS treatment was consistent with changes in Sp1 expression and contrasted the changes observed in miR-130b expression. These findings showed that the miR-130b-Sp1-MUC5AC pathway may play a role in LPS-induced upregulation of MUC5AC in the bile duct epithelium.

Changes in the expression of miR-130b, Spl and MUC5AC in an intrahepatic bile duct stone animal model. To further investigate the miR-130b-Sp1-MUC5AC signaling pathway, an intrahepatic bile duct stone animal model was established
(Fig. 3A). After 15 days of modeling (Fig. 3B), compared with normal rats, the serum total bilirubin and leukocyte indices of the rats in the experiment group significantly increased, which indicated that the biliary tract of the model rats presented inflammation and obstruction caused by pigment stones (Fig. 3C). Bile samples from the model rats were collected after 15 days of modeling. Compared with bile of rats in control group, bile from the rats in experiment group were turbid and formed yellow crystals (Fig. 3D).

RT-qPCR results indicated that the expression of miR-130b was significantly lower in the experimental group compared with the pseudo-operation group (Fig. 3E). Western blotting and RT-qPCR further indicated that compared with the pseudo-operation group, Sp1 protein and mRNA expression in the experimental group were higher (Fig. 3F and G). RT-qPCR results indicated that the mRNA expression of MUC5AC significantly increased in the experimental group compared with the pseudo-operation group (Fig. 3G). Collectively, this series of in vivo experiments suggested that upregulation of MUC5AC expression in intrahepatic bile duct stone tissue was due to expressional changes of miR-130b and Sp1.

Correlation analysis of miR-130b, Sp1 and MUC5AC mRNA expression levels is shown in Fig. $3 \mathrm{H}$ and I. The results indicated that miR-130b and Sp1 mRNA expression levels in bile duct samples showed a significantly negative correlation, whereas mRNA expression levels of Sp1 and MUC5AC showed a significant positive correlation. 
A
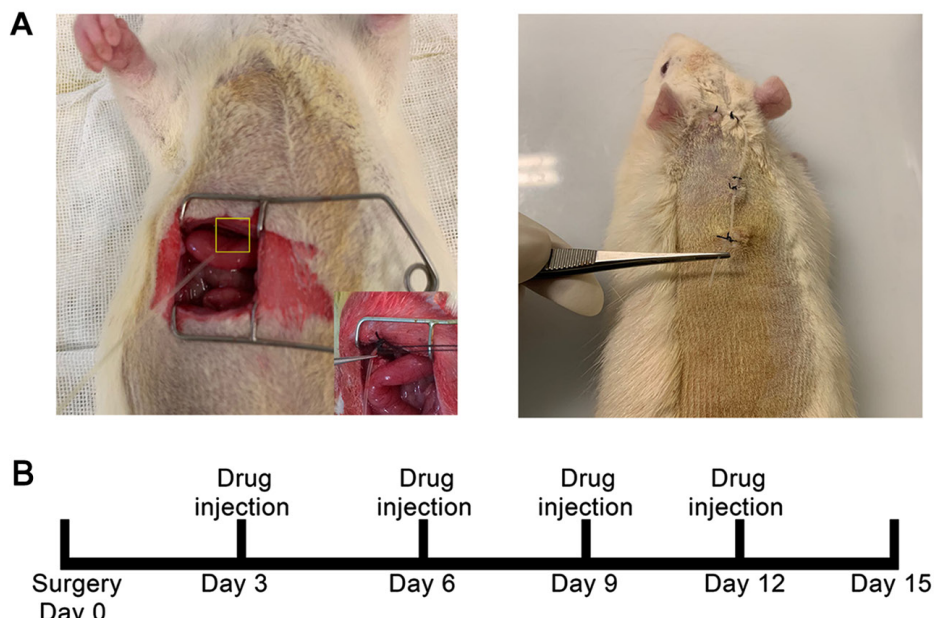

C

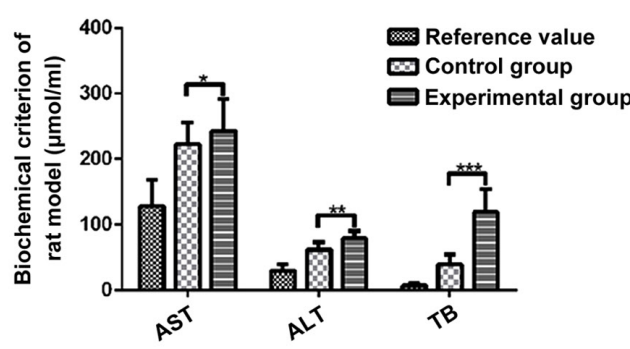

E

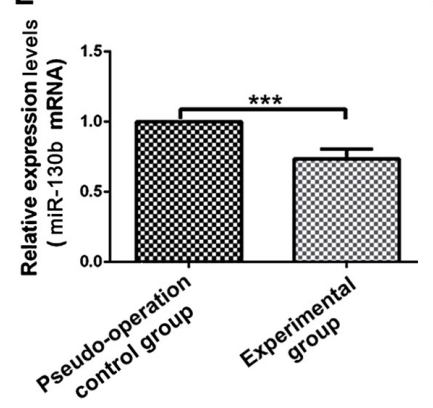

F

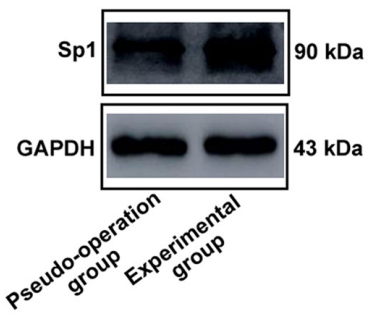

D
G

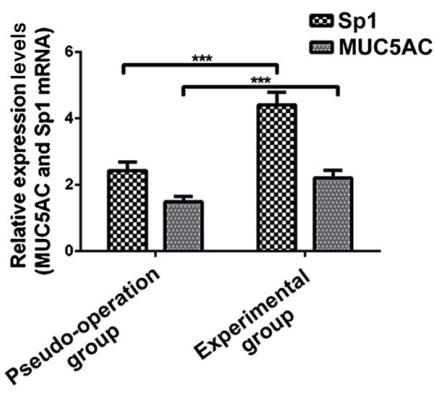

H Relationship between miR-130b and Sp1 Pearson's $r=-0.63 .95 \% \mathrm{Cl}[-0.80,-0.35], P<0.001, n=32$

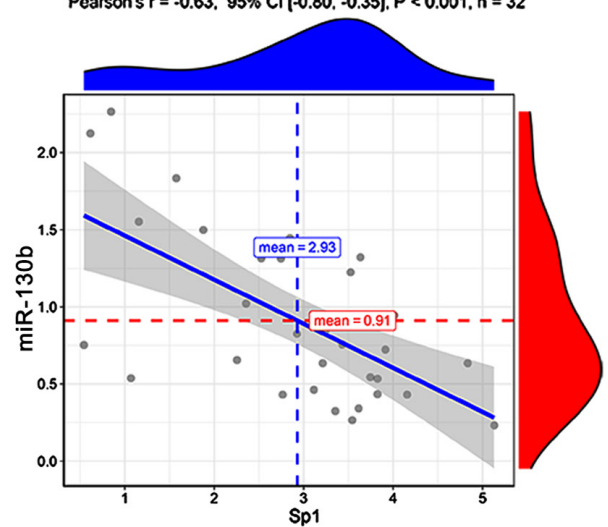

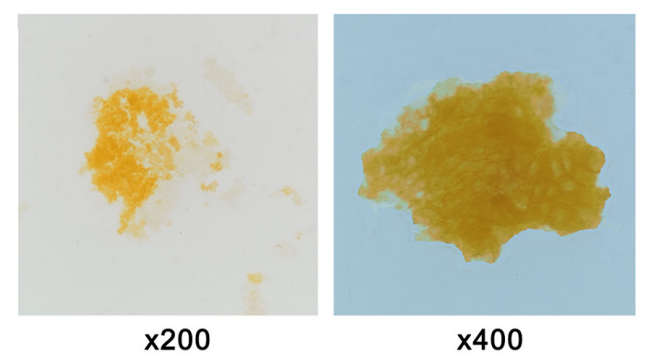

$x 200$ 
A

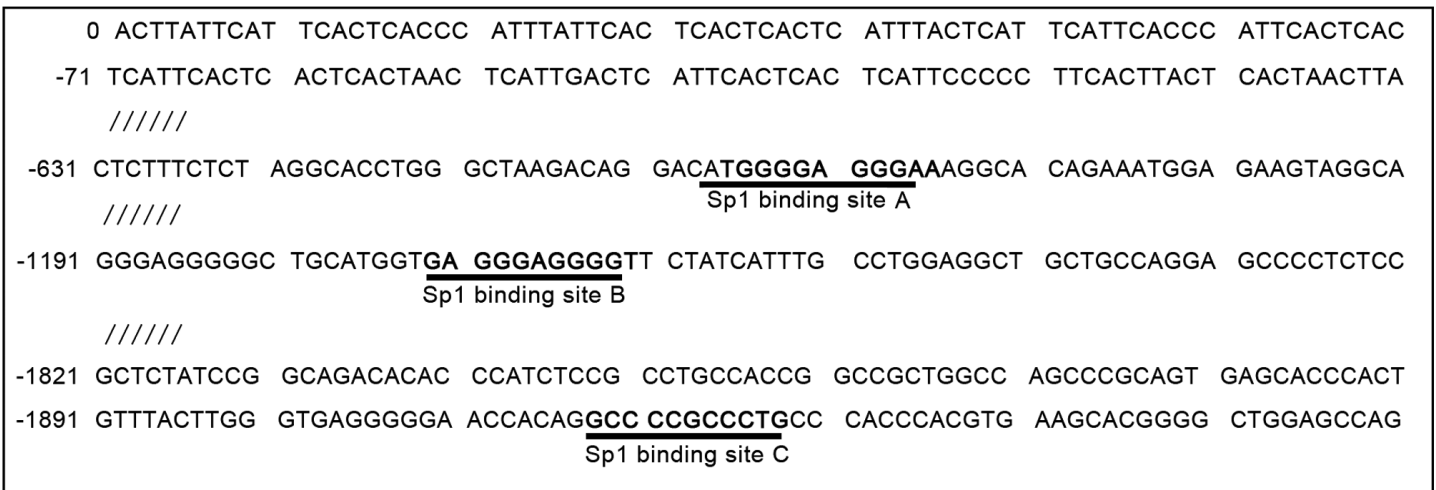

B

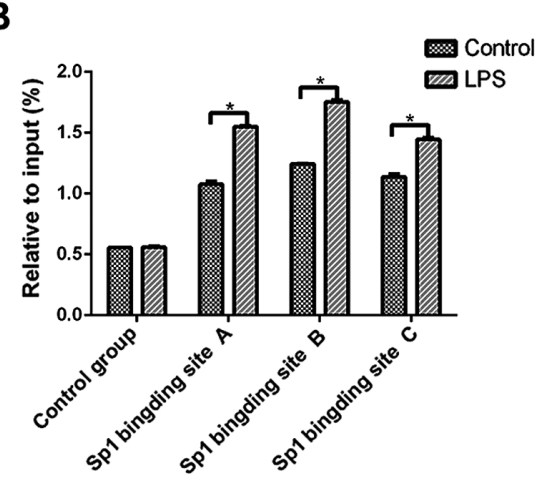

E

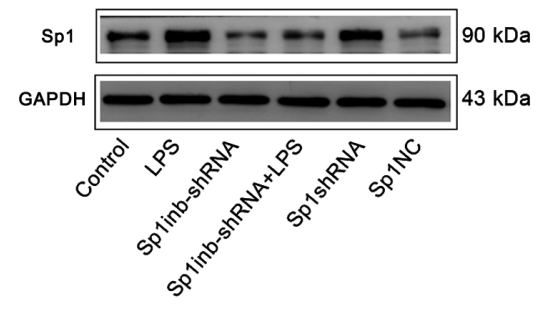

C

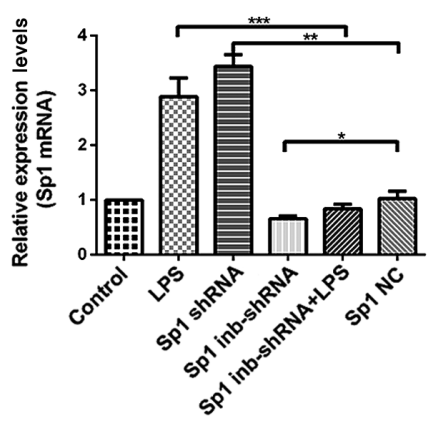

$\mathbf{F}$

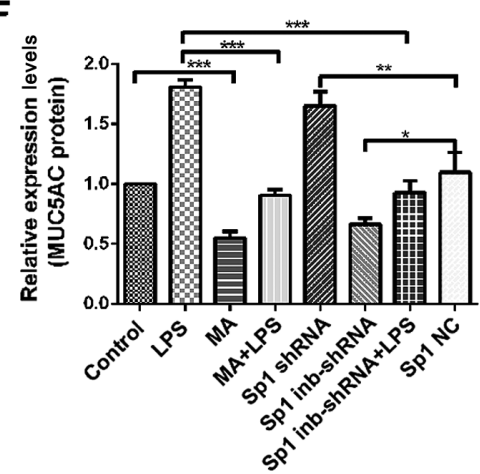

D

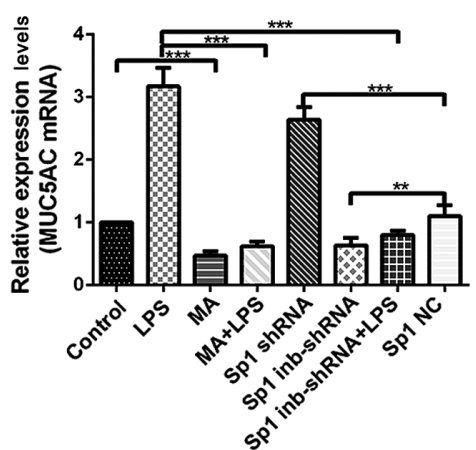

Figure 4. Identifying the direct binding position of Sp1 to the MUC5AC promoter sequence and further verification of the regulatory effect of Sp1 by transfection. (A) An online database was used to predict the possible binding positions for Sp1 to the MUC5AC promoter sequence. (B) Chromatin immunoprecipitation-qPCR experiments showing the Sp1 binding site on the MUC5AC promoter sequence and the alterations of Sp1 binding intensity between control and experimental groups (10 $\mu \mathrm{g} / \mathrm{ml}$ LPS pretreatment for $24 \mathrm{~h}$ ). HIBEpiCs were transfected with shRNA to overexpress Sp1 or shRNA to inhibit Sp1 or pretreated with $10 \mu \mathrm{g} / \mathrm{ml}$ mithramycin A (an Sp1 inhibitor). (C) RT-qPCR detection of Sp1 mRNA expression in HIBEpiCs. (D) Western blotting assay of Sp1 protein expression in HIBEpiCs. (E) RT-qPCR detection of MUC5AC mRNA expression in HIBEpiCs. (F) ELISA detection of MUC5AC expression in HIBEpiC supernatant. ${ }^{*} \mathrm{P}<0.05,{ }^{* *} \mathrm{P}<0.01$ and ${ }^{* * *} \mathrm{P}<0.0001$. MUC5AC, mucin $5 \mathrm{AC}$; Sp1, specificity protein 1 ; HIBEpiCs, human intrahepatic biliary epithelial cells; LPS, lipopolysaccharide; RT-qPCR, reverse transcription-quantitative PCR; shRNA, short hairpin RNA; NC, negative control; MA, mithramycin A.

Sp1 directly regulates MUC5AC expression by binding with its promoter sequence. To verify the direct binding of Sp1 to the MUC5AC promoter sequence and identify the corresponding binding sites, the possible binding sites of Sp1 to the MUC5AC promoter sequences were predicted using online tools. The predictions indicated that there are three possible binding sites, located at positions 665-675, 1209-1219 and 1918-1928 of the MUC5AC promoter sequence (Fig. 4A). Three ChIP-qPCR primers were designed according to these three binding sites (Table II). Cells in the experimental $(10 \mu \mathrm{g} / \mathrm{ml}$ LPS treatment for $24 \mathrm{~h}$ ) and control groups were then evaluated using a ChIP-qPCR assay. All three binding sites played a role in Sp1 binding. The levels Sp1 binding at the three binding sites were significantly higher in the experimental group compared with the control group (Fig. 4B). This indicated that these three binding sites play a role in regulating the effect of $\mathrm{Sp1}$ on MUC5AC expression and that LPS induction significantly increased the levels of Sp1 binding.

To provide further evidence for the regulatory effect of Sp1 on MUC5AC expression, shRNA was used to overexpress Sp1 or suppress Sp1 expression, or cells were pre-treated with MA. RT-qPCR, western blotting and ELISA were then performed to detect the changes in Sp1 and MUC5AC levels. Experimental results showed that Sp1 and MUC5AC mRNA in the overexpression group was significantly higher compared with the negative control group. A marked decline in the Sp1 suppressed 
A

\author{
miR-130b 3' UACGGGAAAGUAGUAACGUGAC 5' \\ Sp1 3'UTR (720-726) 3' GTTTGGGATCAGCTTCTTGCACTC 5' \\ Sp1 3'UTR-mut 3' GTTTGGGATCAGCTTCGGTACAGC 5
}

C

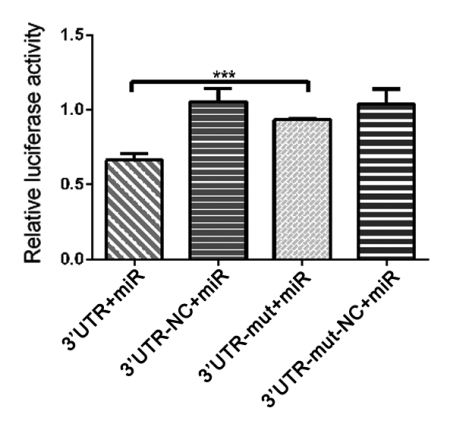

F

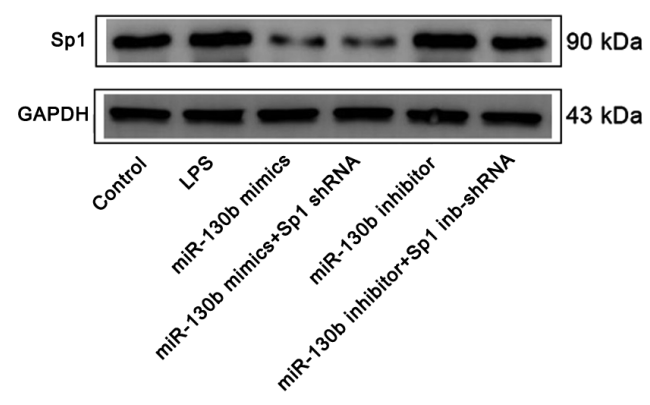

D

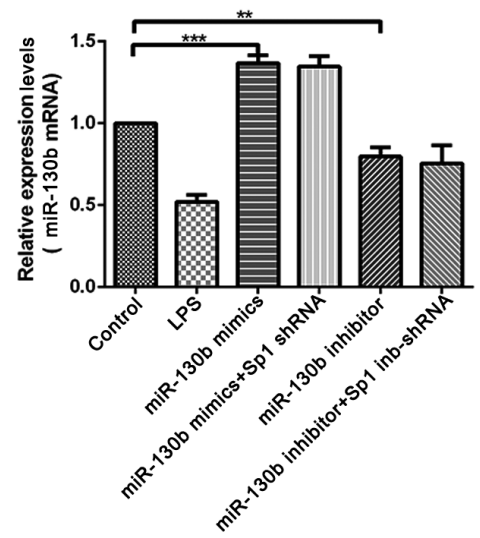

G

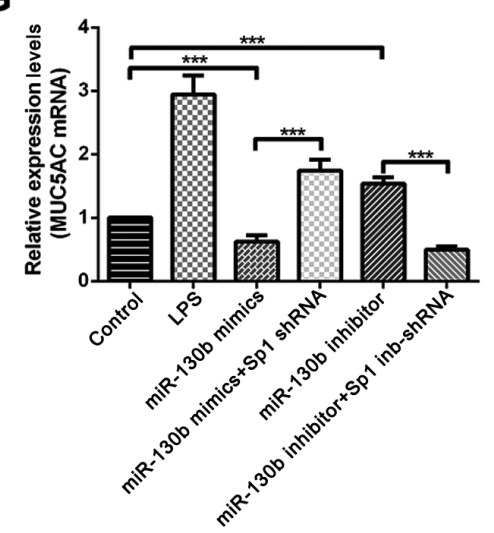

B

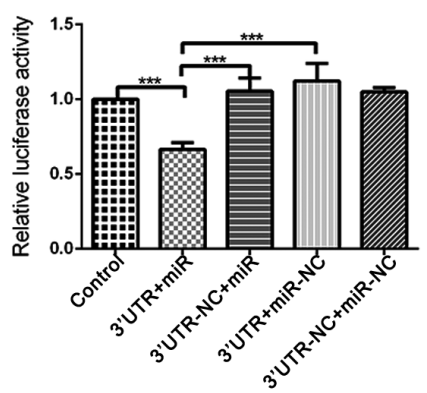

E

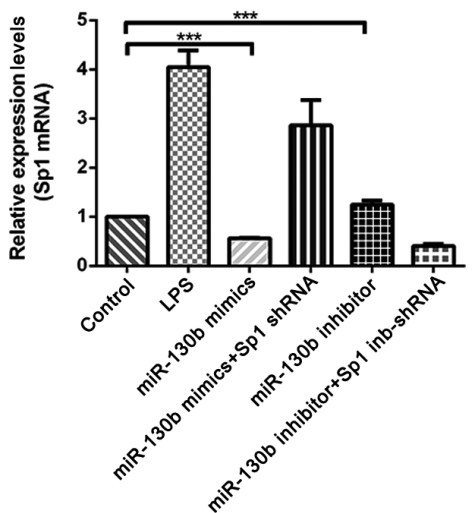

H

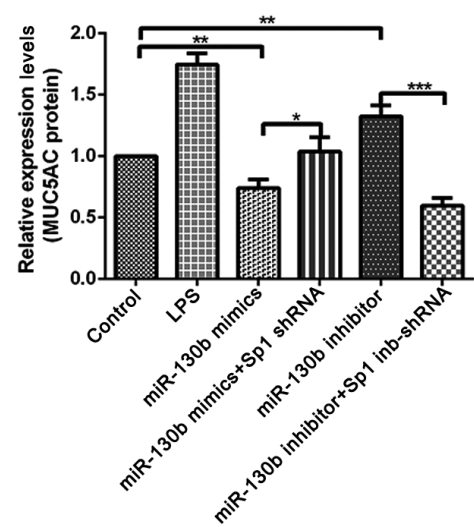

Figure 5. Investigation of the direct binding position between miR-130b and the 3'-UTR sequence of Sp1 and further verification of the regulatory effect of miR-130b on MUC5AC and Sp1 expression. (A) Online tools were used to predict the binding position of miR-130b to the 3'-UTR sequence of Sp1, and the corresponding luciferase primer for a natural plasmid and a mutated plasmid were established. (B and C) Luciferase gene detection for miR-130b and the Sp1 3'-UTR sequence. HIBEpiCs were transfected with miR-130b overexpression mimics or miR-130b inhibitors for 24 h. (D) RT-qPCR detected changes in miR-130b expression in HIBEpiCs. (E) RT-qPCR detected changes in of Sp1 mRNA expression in HIBEpiCs. (F) Western blotting detected changes Sp1 protein expression in HIBEpiCs. (G) RT-qPCR detected changes in of MUC5AC mRNA expression in HIBEpiCs. (H) ELISA detected changes in MUC5AC levels in HIBEpiC supernatant. ${ }^{*} \mathrm{P}<0.05,{ }^{* *} \mathrm{P}<0.01$ and ${ }^{* * *} \mathrm{P}<0.0001$. MUC5AC, mucin 5AC; Sp1, specificity protein 1; miR, microRNA; HIBEpiCs, human intrahepatic biliary epithelial cells; RT-qPCR, reverse transcription-quantitative PCR; 3'-UTR, 3'-untranslated region; LPS, lipopolysaccharide; NC, negative control; mut, mutant; shRNA, short hairpin RNA; inb, inhibitor.

group compared with the negative control group was also observed (Fig. 4C and D). The western blotting results showed that, $\mathrm{Sp1}$ protein expression was significantly increased in the $\mathrm{Sp} 1$ overexpression group, but significantly lower in the Sp1 suppressed-expression group (Fig. 4E). ELISA results showed that compared with their respective controls, MUC5AC levels in the $\mathrm{Spl}$ overexpression group significantly increased, while MUC5AC levels in the MA and Sp1 suppressed-expression group significantly decreased (Fig. 4F).

These results indicated that the effect of LPS-induced MUC5AC upregulation in the bile duct epithelium was regulated by $\mathrm{Sp} 1$. Increase in $\mathrm{Sp} 1$ expression can promote this effect and decreased Sp1 expression can reverse this.
miR-130b directly inhibits Spl expression by binding with its 3'-UTR sequence. Next, the potential binding sites of miR-130b and the Sp1 3'-UTR were predicted using online tools (http://www.targetscan.org/vert_72/). A binding site was found for miR-130b at position 720-726 in the Sp1 3'-UTR sequence (Fig. 5A). Next, luciferase vectors containing full length miR-130b and Sp1 3'-UTR regions were constructed (Fig. 5A), and these luciferase vectors were co-transfected into HIBEpiCs for luciferase reporter gene assays. The results indicated that, compared with the negative control group, the fluorescence intensity of the 3'UTR+mir group significantly decreased (Fig. 5B), thus showing that miR-130b binds directly to the Sp1 3'-UTR sequences. A point mutation vector for miR-130b was 
constructed (Fig. 5A), and this was co-transfected with the Sp1 3'-UTR fragment into HIBEpiCs. It was found that the fluorescence intensity of $3^{\prime} \mathrm{UTR}$-nu+miR group relatively increased compared with 3'UTR+mir group (Fig. 5C).

To verify the suppressive effect of miR-130b on the expression of Sp1, a miR-130b overexpression mimic and an inhibitor-expression plasmid were transfected into HIBEpiCs for a functional recovery assay, and the expression levels of miR-130b, Sp1 and MUC5AC were detected by western blotting, RT-qPCR and ELISA. RT-qPCR results for miR-130b detection showed that, compared with the control group, the expression of miR-130b significantly increased in the miR-130b mimics group, while the expression significantly decreased in the inhibitor group (Fig. 5D). RT-qPCR and western blotting results further showed that the mRNA and protein expression of $\mathrm{Sp} 1$ decreased in the miR-130b mimics group, and increased in the miR-130b inhibitor group, when compared with the control group (Fig. 5E and F). ELISA and RT-qPCR results further showed that compared with the control group, the mRNA and protein expression of MUC5AC in the miR-130b mimics group was significantly lower, but significantly higher in the miR-130b inhibitor group. In addition, the increase of MUC5AC induced by Sp1 shRNA can be suppressed by miR-130b mimics (Fig. 5G and H).

These results indicated that LPS-induced MUC5AC upregulation was achieved by increasing Sp1 expression and Sp1 expression was directly regulated by miR-130b. Hence, the miR-130b-Sp1-MUC5AC signaling pathway may play a role in the development of intrahepatic bile stones.

\section{Discussion}

Intrahepatic cholelithiasis is a common disease in the biliary system among the Chinese population (1). At present, the surgical and clinical treatment method of intrahepatic cholelithiasis is relatively limited in hepatectomy or choledochoscopic lithotripsy, however, these method were accompanied with numerous complications such as intrahepatic insufficiency and recurrence of calculus after treatment (27). Therefore, it is of importance to study the detailed mechanism of the disease and develop new treatment methods. Previous studies showed that LPS produced by bacterial infection of bile duct could increase MUC5AC secretion in the bile duct epithelium, which then promotes bile duct stone formation $(3,6)$. However, to the best of our knowledge, the mechanism of MUC5AC overexpression following bacterial infection has not been elucidated. The present study showed that MUC5AC and Sp1 expression in the bile duct tissue of patients with hepatolithiasis was higher compared with the control group, while the expression of miR-130b was relatively low. In addition, LPS-treated bile duct epithelial cells and in vivo experiments of Sprague-Dawley rats showed similar results. Therefore, it was concluded there may be a mutual regulation between miR_130b, Sp1 and MUC5AC secretion. ChIP and luciferase reporter assays confirmed that miR-130b has one binding site in the 3'-UTR region of Sp1, which may regulate $\mathrm{Sp1}$ expression by binding to this site. Spl contains three binding sites in the promoter sequence of MUC5AC, which may regulate MUC5AC secretion when Sp1 binding with these three sites $(15,18)$. In cell transfection experiments, it was found that the expression of Sp1 and MUC5AC decreased when miR-130b was overexpressed, while inhibition of miR-130b expression could promote the secretion of Sp-1 and MUC5AC. Similarly, in the case Sp1 overexpression, MUC5AC secretion increased, while inhibition of Spl expression resulted in decreased MUC5AC expression. This further confirmed the regulatory effects of miR-130b to Sp1 and Sp1 to MUC5AC. Therefore, the present study concluded that miR-130b and Sp1 may play a role in MUC5AC overexpression in the intrahepatic bile duct epithelium caused by bacterial infection. Utilizing the regulatory effect of miR-130b and Sp1 to promote or inhibit miR-130b and Sp1 expression may provide a new strategy and method for the treatment of hepatolithiasis. Of note, MUC5AC overexpression is not the only factor responsible for intrahepatic bile duct stone formation. Although influencing miR-130b and Sp1 expression can partly regulate MUC5AC secretion, it may not completely avoid stone formation. Therefore, how to effectively use miR-130b, Sp1 and MUC5AC as a signal transduction pathway for the treatment of hepatolithiasis still needs more further experimental research.

\section{Acknowledgements}

Not applicable.

\section{Funding}

This study was funded by the Outstanding Scientific Fund of Shengjing Hospital (grant no. A250) and the National Natural Science Foundation of China (grant no. A376).

\section{Availability of data and materials}

The datasets used and/or analyzed during the current study are available from the corresponding author on reasonable request.

\section{Authors' contributions}

SW and JK designed the study. XW, ZZ, CY and JH performed the experiments. XW, YF and YT contributed to data collection, statistical analysis, data interpretation and manuscript preparation. All authors read and approved the final manuscript.

\section{Ethics approval and consent to participate}

The study was approved by the local ethics committee of the Affiliated Shengjing Hospital of China Medical University (approval no. 2017PS231K).

\section{Patient consent for publication}

Not applicable.

\section{Competing interests}

The authors declare that they have no competing interests.

\section{References}

1. Kim HJ, Kim JS, Joo MK, Lee BJ, Kim JH, Yeon JE, Park JJ, Byun KS and Bak YT: Hepatolithiasis and intrahepatic cholangiocarcinoma: A review. World J Gastroenterol 21: 13418-13431, 2015. 
2. Kim HJ, Kim SH, Chae GB, Lee SJ and Kang CD: Increased expression of mucin 5AC mRNA and decreased expression of epidermal growth-factor receptor mRNA in gallstone patients. Tohoku J Exp Med 214: 139-144, 2008

3. Wu SD, Yu H and Sun JM: Bacteriological and electron microscopic examination of primary intrahepatic stones. Hepatobiliary Pancreat Dis Int 5: 228-231, 2006.

4. Yoo KS, Choi HS, Jun DW, Lee HL, Lee OY, Yoon BC, Lee KG, Paik SS, Kim YS and Lee J: MUC Expression in gallbladder epithelial tissues in cholesterol-associated gallbladder disease. Gut Liver 10: 851-858, 2016.

5. Chuang SC, His E and Lee KT: Mucin genes in gallstone disease Clin Chim Acta 413: 1466-1471, 2012.

6. Li M, Tian Y, Wu S, Yu H and Li Y: LPS stimulates MUC5AC expression in human biliary epithelial cells: Whether there exists a possible pathway of PKC/NADPH/ROS? Mol Cell Biochem 385: 87-93, 2014.

7. Tan NY and Khachigian LM: Sp1 phosphorylation and its regulation of gene transcription. Mol Cell Biol 29: 2483-2488, 2009.

8. Baños-Lara Mdel R, Piao B and Guerrero-Plata A: Differential mucin expression by respiratory syncytial virus and human metapneumovirus infection in human epithelial cells. Mediators Inflamm 2015: 347292, 2015

9. Zen Y, Harada K, Sasaki M, Tsuneyama K, Katayanagi K, Yamamoto Y and Nakanuma Y: Lipopolysaccharide induces overexpression of MUC2 and MUC5AC in cultured biliary epithelial cells: Possible key phenomenon of hepatolithiasis. Am J Pathol 161: 1475-1484, 2002.

10. Vilkin A, Nudelman I, Morgenstern S, Geller A, Dayan YB Levi Z, Rodionov G, Hardy B, Konikoff F, Gobbic D and Niv Y: Gallbladder inflammation is associated with increase in mucin expression and pigmented stone formation. Dig Dis Sci 52: $1613-1620,2007$.

11. Corfield AP, Myerscough N, Longman R, Sylvester P, Arul S and Pignatelli M: Mucins and mucosal protection in the gastrointestinal tract: New prospects for mucins in the pathology of gastrointestinal disease. Gut 47: 589-594, 2000.

12. Ma J, Rubin BK and Voynow JA: Mucins, mucus and goblet cells. Chest 154: 169-176, 2017.

13. Ali MS and Pearson JP: Upper airway mucin gene expression: A review. Laryngoscope 117: 932-938, 2010.

14. Rose MC and Voynow JA: Respiratory tract mucin genes and mucin glycoproteins in health and disease. Physiol Rev 86 : 245-278, 2006

15. Hewson CA, Edbrooke MR and Johnston SL: PMA induces the MUC5AC respiratory mucin in human bronchial epithelial cells, via PKC, EGF/TGF-alpha, Ras/Raf, MEK, ERK and Sp-1-dependent mechanisms. J Mol Biol 344: 683-695, 2004
16. Huang $\mathrm{C}$ and Xie K: Crosstalk of $\mathrm{Sp} 1$ and Stat 3 signaling in pancreatic cancer pathogenesis. Cytokine Growth Factor Rev 23: $25-35,2012$

17. Jiang W, Jin Z, Zhou F, Cui J, Wang L and Wang L: High co-expression of Sp1 and HER-2 is correlated with poor prognosis of gastric cancer patients. Surg Oncol 24: 220-225, 2015.

18. Di YP, Zhao J and Harper R: Cigarette smoke induces MUC5AC protein expression through the activation of Sp1. J Biol Chem 287: 27948-27958, 2012.

19. Oyanagi T, Takizawa T, Aizawa A, Solongo O, Yagi H, Nishida Y Koyama H, Saitoh A and Arakawa H: Suppression of MUC5AC expression in human bronchial epithelial cells by interferon- $\gamma$. Allergol Int 66: 75-82, 2016.

20. Aslam F, Palumbo L, Augenlicht LH and Velcich A: The Sp family of transcription factors in the regulation of the human and mouse MUC2 gene promoters. Cancer Res 61: 570-576, 2001.

21. Luke B and David E: Airway mucus and asthma: The role of MUC5AC and MUC5B. J Clin Med 6: 112, 2017.

22. Zheng H, Dong X, Liu N, Xia W, Zhou L, Chen X, Yang Z and Chen X: Regulation and mechanism of mouse miR-130a/b in metabolism-related inflammation. Int J Biochem Cell Biol 74: 72-83, 2016

23. Li L, Gao F, Jiang Y, Yu L, Zhou Y, Zheng H, Tong W, Yang S, Xia T, Qu Z and Tong G: Cellular miR-130b inhibits replication of porcine reproductive and respiratory syndrome virus in vitro and in vivo. Sci Rep 5: 17010, 2015.

24. Kaur K, Bhatia H and Datta M: MicroRNAs in hepatic pathophysiology in diabetes. World J Diabetes 2: 158-163, 2011.

25. Contreras $\mathbf{J}$ and Rao DS: MicroRNAs in inflammation and immune responses. Leukemia 26: 404-413, 2012.

26. Kim C, Lee H, Cho YM, Kwon OJ, Kim W and Lee EK: TNFalpha-induced miR-130 resulted in adipocyte dysfunction during obesity-related inflammation. FEBS Lett 587: 3853-3858, 2013.

27. Tazuma S, Unno M, Igarashi Y, Inui K, Uchiyama K, Kai M, Tsuyuguchi T, Maguchi $\mathrm{H}$, Mori $\mathrm{T}$, Yamaguchi $\mathrm{K}$, et al: Evidence-based clinical practice guidelines for cholelithiasis 2016. J Gastroenterol 52: 276-300, 2017.

This work is licensed under a Creative Commons Attribution-NonCommercial-NoDerivatives 4.0 International (CC BY-NC-ND 4.0) License. 